\title{
LESSONS LEARNED FROM THE JEFFERSON LAB - SNS CRYOMODULE PRODUCTION RUN
}

\author{
J. Hogan ${ }^{\dagger}$, E. F. Daly, J. Fischer, J. Preble \\ Thomas Jefferson National Accelerator Facility \\ Newport News, VA 23606, USA
}

\begin{abstract}
In light of the recent developments with the International Linear Collider (ILC), and the recommendation to utilize "Cold" technology for this future particle accelerator, this paper will present the lessons learned from the recently concluded Spallation Neutron Source (SNS) superconducting radio frequency (SRF) cryomodule production run at the Thomas Jefferson National Accelerator Facility (Jefferson Lab). Over the past twenty years Jefferson Lab has worked with industry to successfully design, manufacture, test and commission more SRF cryomodules than any other entity in the United States. The knowledge gained from the design and fabrication of the SNS prototype, eleven - 0.61 (medium) beta and the twelve - 0.81 (high) beta cryomodules, will prove to be an effective asset to the ILC project. After delivery of the final production cryomodule in March 2005, design and fabrication data will be collected, evaluated and presented to make this information beneficial for future particle accelerator projects. Recommendations with respect to these findings will also be presented as an integral part of this paper.
\end{abstract}

KEYWORDS: Accelerator, Cryomodule, SRF, Cavity, SNS, JLAB

PACS: $29.17 .+w$ Electrostatic, collective, and linear accelerators

\section{INTRODUCTION}

The superconducting portion of the SNS linear accelerator consists of eleven medium beta cryomodules and twelve high beta cryomodules housing thirty-three $\beta=0.61$ and forty-eight $\beta=0.81 \mathrm{SRF}$ cavities, respectively[1]. These cryomodules are designed to thermally maintain the SRF cavities at $2.1 \mathrm{~K}$ continuously for several years [2]. A secondary helium cooling circuit is incorporated into the cryomodule design to maintain a 5-6 K temperature on the outer conductor cold flange portion of the fundamental power coupler. The shield circuit is designed to operate at $35-55 \mathrm{~K}$ and effectively act as a radiant shield and a thermal anchor for all components that have a direct conduction path between room temperature and the cold mass. This paper will focus on the cryogenic applications of various cryomodule components and subsystems, their performance and the lessons learned from the assembly and testing processes. 


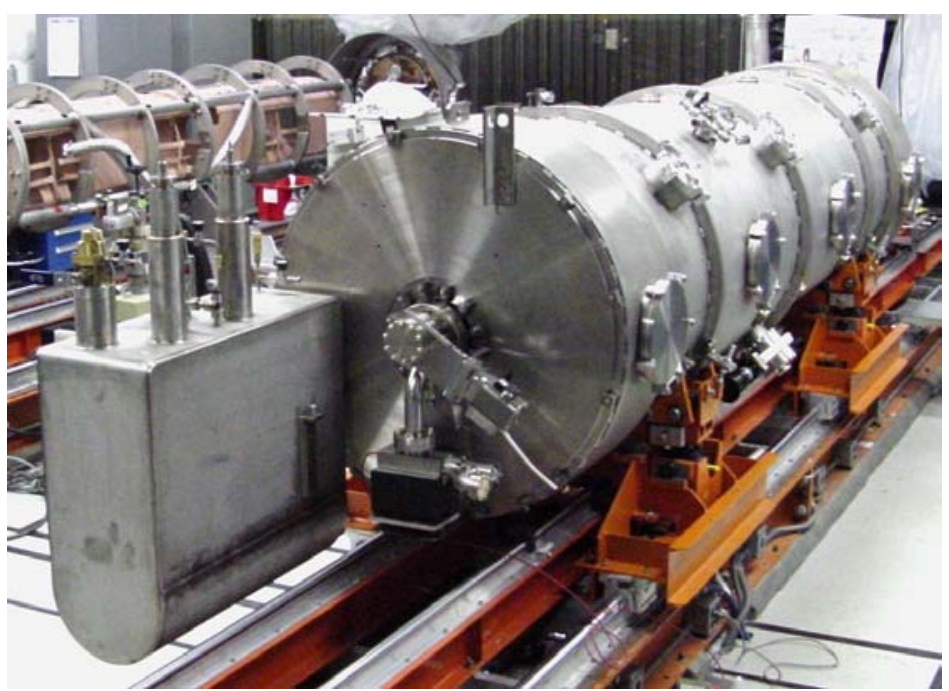

FIGURE 1. SNS Cryogenic Supply End Assembly Installed onto a Medium Beta Cryomodule

\section{CRYOGENIC END CANS}

All of the cryogenic fluid flow through the SNS cryomodules is handled via a supply and return cryogenic end can assembly (FIGURE 1). The ability to accurately monitor the properties of the primary low pressure helium circuit throughout the cryomodule is critical to its function. Two pressure transducers are utilized on the primary low pressure circuit for the SNS cryomodule and are located within the end cans. The first is a broad range (05000 torr) transducer for initial cooldown. The second is a small range (0-100 torr) transducer providing a higher resolution for transition through lambda and steady state operations. The pressure transducer instrumentation is mounted external to the end cans at room temperature and is easily maintained. The pressure transducer for the secondary coupler circuit is designed and functions in a similar manner.

The instrumentation used to monitor the temperature of the helium flow through the primary circuit for the SNS cryogenic end cans proved to be more problematic than originally anticipated. The prototype set of end cans were put through an extensive quality assurance (QA) program after receipt by JLab. One portion of this QA program was to rapidly thermal cycle the internal piping circuits (primary, secondary \& shield) to LN2 temperature and back to room temperature three consecutive times. Following this "cold shock" procedure all circuits were leak checked with a helium mass spectrometer to a minimum detectable leak (MDL) rate of less than $2 \times 10^{-10} \mathrm{~atm} \mathrm{cc} / \mathrm{sec}$. This first set of end cans passed these QA procedures as prescribed by the project requirements. Based on these positive results, early production end can assemblies were leak checked with a helium mass spectrometer but were not LN2 "cold shocked" prior to being put into cryomodule production.

Once each cryomodule was completely assembled, a portion of them were cryogenically tested with $\mathrm{rf}$ power at $2.1 \mathrm{~K}$ in order to qualify the performance of the cavities and all of the supporting cryomodule subsystems. During this "cold" cryomodule testing procedure, a leak was discovered in one of the cryogenic end can temperature feedthroughs. Based on this failure mode, all remaining cryogenic end can assemblies were cycled three times through LN2 and room temperature, followed by a helium leak check prior to being assembled onto a cryomodule. A total of four end cans were repaired at JLab during SNS cryomodule production run. 


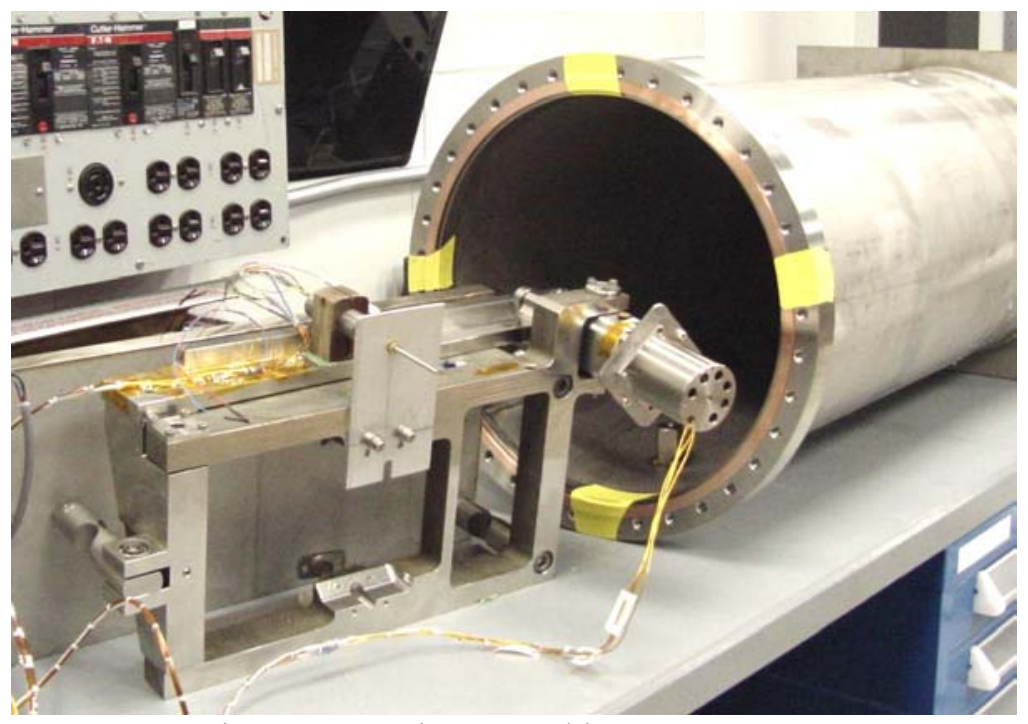

FIGURE 2. SNS Prototype Cavity Tuner Testing Assembly

This additional cold shocking procedure was intended to identify and eliminate any future leaks related to the end can temperature diode feedthroughs. Confidence in this proactive approach was supported by the fact that all of the end can assemblies that were LN2 cold shocked and tested at $2.1 \mathrm{~K}$ as part of the cryomodule testing procedure did not have any leaks. Additional leaks have been discovered in the end cans after cryomodule delivery to SNS [3].

\section{CAVITY FREQUENCY TUNERS}

The requirement of the cavity tuners is to set and maintain the operational frequency of the SRF cavities within a $500 \mathrm{kHz}$ range and a $10 \mathrm{~Hz}$ resolution (FIGURE 2). The SNS cavity tuner design is based on an early version of the TESLA/SACLAY cavity tuner. As with the end can assemblies, the first cavity tuner assembly was put through an extensive QA procedure that included performance measurement within its actual operational environment. The cavity tuners are conductively anchored to the helium vessel and are required to operate within the insulating vacuum space. The complete tuner assembly design was optimized through this prototyping/QA process and the final system was specified and released for production.

During cold $\mathrm{rf}$ testing of the first production medium beta cryomodule, the tuner assemblies failed. After investigation, it was discovered that the vendor supplying the drive mechanisms had changed one of the internal parts from the first assemblies delivered to JLab for qualification. The result was that the tuner production parts were not manufactured to the same specification as the tuner prototyping parts. Once this discrepancy was brought to the attention of the vendor, all parts were returned to the vendor and reworked as per the original specification. These follow up activities have resulted in this cavity tuner failure mode being eliminated from all SNS cryomodules. 


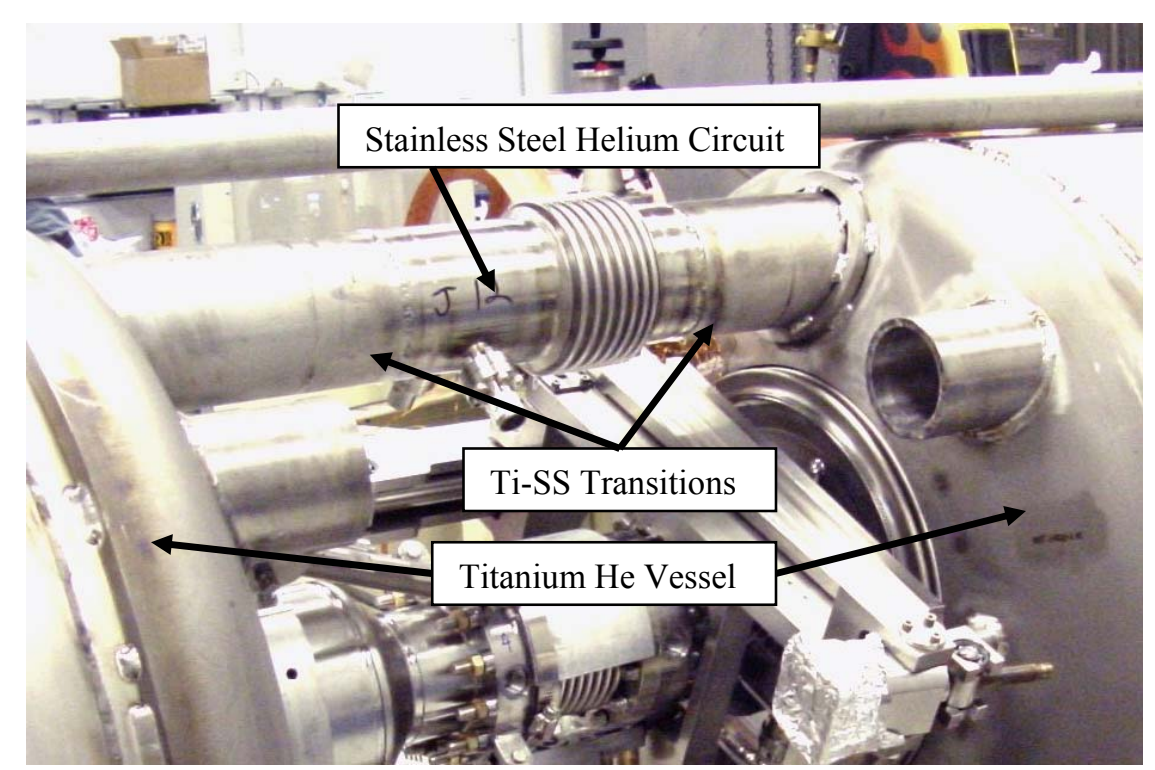

FIGURE 3. Titanium-to-Stainless Steel Explosion Bond Transition Welded into Helium Circuit

\section{TITANIUM-STAINLESS STEEL TRANSISTIONS}

The titanium to stainless steel explosion bonded joint is used to connect the titanium helium pressure vessels to the stainless steel primary process piping (FIGURE 3). This material transitional concept has been used on the CEBAF Upgrade cryomodule with notable success [4]. Before any explosion bonded joint is released for use in cryomodule production; $100 \%$ of all parts are LN2 cold shocked three times and leak checked with a helium mass spectrometer.

The primary failure mode associated with this explosion bonded joint design is related to the thermal stresses induced during the welding process. Each explosion bonded joint must go through two welding cycles, one to titanium and the other to stainless steel. Early in the SNS cryomodule production run, a small number of explosion bonded joints began to fail during the second welding procedure. During the investigation into this failure mode, it was noted the first weld procedure was performed by the helium vessel vendor and the joint was leak tight upon receipt at JLab. Additionally, the vendor supplying the explosion bonded transition joints had developed a new method for manufacturing the titanium to stainless steel transitions that promised to be more robust.

JLab decided to develop a specific welding procedure that would control the amount of thermal stress introduced from the welding to the explosion bonded joints. By providing active cooling around the explosion bond during the welding process, JLab was able to maintain the integrity of the transition joint. This new procedure was used in conjunction with the new transition joint for the majority of the SNS cryomodule production. In addition, JLab decided to take full control of the explosion bonded joint welding process by removing this welding requirement from the helium vessel vendor. From this point forward, JLab maintained full control of the process by performing both welding procedures on all explosion bonded joints. It is worth noting all explosion bonded joints are LN2 cold shocked and leak checked with a helium mass spectrometer after all welding has been completed. 


\section{CONCLUSION}

From an industrial management standpoint, all of these lessons learned may be categorized as quality control and/or process control. However, the intent of this paper is to focus on the importance of the QA function with respect to suppliers. The relationship with suppliers will have a significant impact on both cost and schedule for cryomodule production. The SNS cryomodule production run consisted of a total of twenty-three cryomodules manufactured over a two year time frame. The peak production rate accomplished by JLab during this production run was a cryomodule being completed every four weeks. For a project the size and scope of the ILC, the cryomodule production rate will have to be on the order of ten times the SNS production rate. Therefore, the cryomodule production run for the ILC must work with the suppliers to ensure the cryogenic subsystems and processes have been thoroughly vetted for function and reliability. Furthermore, several prototype cryomodules should be completely built and thoroughly tested, as a means to verify all subsystems properly function together as a whole, under real environmental operating conditions.

\section{ACKNOWLEDGEMENTS}

Work supported by the U.S. Department of Energy under contract DE-AC05-00OR22725

\section{REFERENCES}

1. W. J. Schneider et al., "Design of the Spallation Neutron Source (SNS) Cryomodule", PAC2001, Chicago, IL June 2001.

2. Daly, E. F. et al., "Thermal Performance of the SNS Cryomodule" in Advances in Cryogenic Engineering 49A, edited by Joseph Waynert et al., Plenum, New York, 2000, pp. 531-537.

3. E. I. Campisi et al., "Testing of the SNS Superconducting Cavities and Cryomodules", PAC2005, Knoxville, TN May 2005.

4. J. P. Hogan et al., "Design of the CEBAF Energy Upgrade Cryomodule Cold Mass" PAC2001, Chicago, IL June 2001 